\title{
Experimental Estimation on Magnetic Friction of Superconductor Flywheel Energy Storage System
}

\author{
Jeong-Phil Lee*, Sang-Chul Han, and Byeong-Choel Park \\ Korea Electric Power Research Institute, 103-61 Munji-Dong Yuseong-Gu, Daejeon, Korea
}

(Received 15 March 2011, Received in final form 20 May 2011, Accepted 17 June 2011)

\begin{abstract}
This study estimated experimentally the loss distribution caused by magnetic friction in magnetic parts of a superconductor flywheel energy storage system (SFES) to obtain information for the design of high efficiency SFES. Through the spin down experiment using the manufactured vertical shaft type SFES with a journal type superconductor magnetic bearing (SMB), the coefficients of friction by the SMB, the stator core of permanent magnet synchronous motor/generator (PMSM/G), and the leakage flux of the metal parts were calculated. The coefficients of friction by the stator core of PMSM/G in case of using Si-steel and an amorphous core were calculated. The energy loss by magnetic friction in the stator core of PMSM/G was much larger than that in the other parts. The level of friction loss could be reduced dramatically using an amorphous core. Energy loss by the leakage magnetic field was small. On the other hand, the energy loss could be increased under other conditions according to the type of metal nearby the leakage magnetic fields. In manufactured SFES, the rotational loss by the amorphous core was approximately 2 times the loss of the superconductor and leakage. Moreover, the rotational loss by the Si-steel core is approximately $3 \sim 3.5$ times the loss of superconductor and leakage.
\end{abstract}

Keywords : magnetic friction, superconductor, flywheel, amorphous core, rotational loss

\section{Introduction}

The Superconductor Flywheel Energy Storage System (SFES) is an electric power storage system in which the electrical energy is stored by converting it into mechanical rotational energy. The SFES using a superconductor magnetic bearing (SMB) $[1,2]$ is a environment-friendly utility without chemicals and has higher energy storage efficiency than other type flywheels using a mechanical ball bearing and a magnetic bearing. The energy loss characteristics and efficiency of a SFES using SMB have been evaluated in many studies [3-6]. The SFES shows a small amount of loss caused by the inhomogeneous magnetic field of a superconductor. On the other hand, energy loss by magnetic friction during rotation is increased because the SFES has magnetic fields in some parts, such as the SMB $[4,7]$ permanent Magnet type Synchronous motor/generator $(\mathrm{PMSM} / \mathrm{G})$ [8], and windage loss depending on the degree of vacuum. Since the energy loss at the degrees of vacuum under $1 \mathrm{E}^{-4}$ [torr] can be ignored [2], the total energy loss of the SFES can be reduced

*Corresponding author: Tel: +82-42-865-5917

Fax: +82-42-865-5917, e-mail: jplee@kepri.re.kr significantly if the losses by the SMB and PMSM/G can be reduced [8]. Therefore, the loss mechanism caused by various parts of the SFES needs to be assessed.

In this paper, to obtain energy loss information to develop the high energy efficiency SFES, the magnetic friction in some parts of the SFES with magnetic fields was estimated experimentally by obtaining the coefficient of friction through a spin down experiment of the SFES. A vertical shaft type SFES was manufactured with a journal type SMB. The coefficients of friction by the $\mathrm{SMB}$, the stator core of PMSM/G, and metal parts by the leakage flux were calculated using the data obtaining from the spin down experiment. In particular, the coefficients of friction for the stator core of the PMSM/G in the case of using Si-steel and an amorphous core was calculated. From the results, the energy loss by magnetic friction in the stator core of PMSM/G was much larger than that in the other parts. The friction loss can be reduced dramatically using an amorphous core. The energy loss by the leakage magnetic field is small. On the other hand, energy loss can be increased under other conditions according to the type of metal nearby the leakage magnetic fields. 


\section{Design of a SFES}

The SFES is composed of a flywheel for storing energy, a SMB for levitating the wheel, which is composed of superconductors and permanent magnets, and a motor/ generator for energy conversion. Fig. 1 shows a photograph of the manufactured SFES for assessing the energy loss characteristics.

In this paper, the SFES system was constructed using a journal type SMB enclosing the flywheel axis, as shown in Fig. 1. This type has a powerful levitation force and stability by supporting the axis in the direction of the centrifugal force. As shown in Fig. 1, the SMB is located at the upper part and lower part of the flywheel. The SMB is composed of superconductors and permanent magnets. Six YBCO bulk superconductors with dimensions of $30 \times 25 \times 10 \mathrm{~mm}$ were used. The permanent

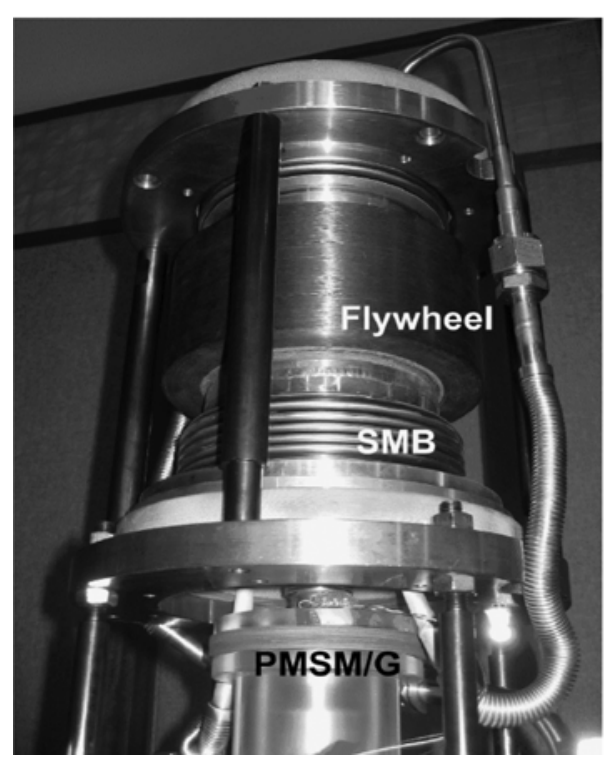

Fig. 1. Photograph of the manufactured SFES system.

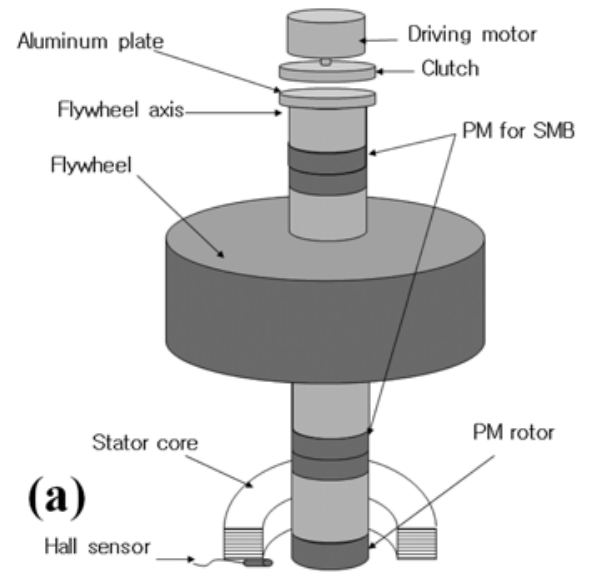

magnet for the SMB has a $10 \mathrm{~mm}$ inner radius, $20 \mathrm{~mm}$ outer radius and a thickness of $10 \mathrm{~mm}$. The flywheel for energy storage is composed of a titanium shaft and a composite wheel. The wheel length, diameter and total weight is $500 \mathrm{~mm}, 120 \mathrm{~mm}$ and $4 \mathrm{~kg}$, respectively.

The stator core and PM rotor of the PMSM/G are in the lower part of the SFES. Fig. 2 shows the slotless type core used for assessing the losses according to the core size variation. The stack height of the stator core was 10 $\mathrm{mm}$. The installed NdFeB35 series PM for PMSM/G was magnetized in the radial direction, resulting in 2 poles. The inner radius, outer radius, thickness and residual flux density of the magnet was $10 \mathrm{~mm}, 20 \mathrm{~mm}, 10 \mathrm{~mm}$ and $1.23 \mathrm{~T}$, respectively. All parts were installed in a vacuum chamber to avoid windage loss by air friction during rotation.

\section{Measurement}

The experiments were performed in a chamber that was evacuated using a molecular turbo pump. The pressure in the chamber was $<10^{-5}$ torr to prevent loss by air friction. The wheel center was adjusted after elevating the wheel to the field cooling position using upper and lower pivots. The YBCO bulks were cooled down using circulating liquid nitrogen (LN2). After the temperature of the SMB decreased to $77 \mathrm{~K}$, the lower and upper part pivots were removed. After levitating wheel, the wheel was rotated to more than 6,000 RPM using a motor. An eddy current clutch was used to accelerate the wheel. The clutch consisted of an aluminum plate connected to the top of the wheel shaft and an aluminum plate in which the PMs were embedded. The clutch was located outside of the vacuum chamber and driven by an electric motor. After turning off the motor, the naturally decreasing rotational speed according to time under some magnetic friction

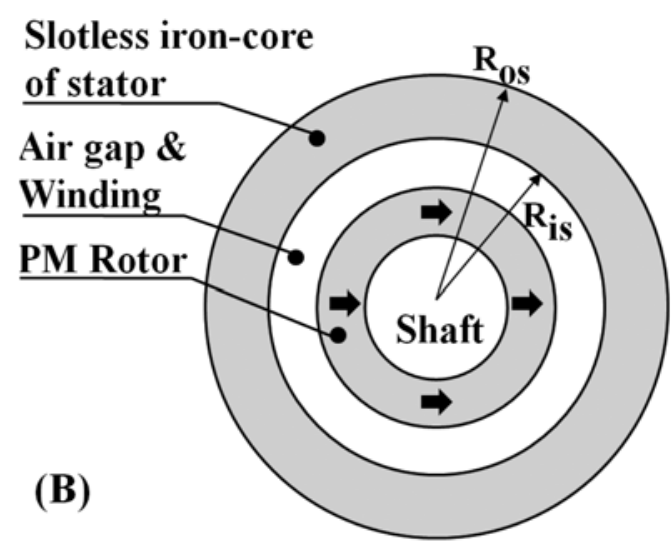

Fig. 2. Structure of the SFES (a) flywheel structure and (b) rotor and stator. 
conditions was measured. The method for measuring the decreasing rotational speed employed a hall sensor installed in the lower part of Fig. 2(a)

First, to assess the energy loss by the SMB, after the stator core and PM rotor of PMSM/G were removed, the pressure in the chamber was maintained at $<10^{-5}$ torr to eliminate energy loss due to air friction. Since there was no air friction loss due to high degree of vacuum, the energy loss was caused by the SMB. The losses caused by leakage flux were included in this measured loss. A rotational core type PMSM with the same core was used to obtain only the losses caused by leakage flux because a rotational core type PMSM has only losses by leakage flux without any loss produced by the core. A rotational core type PMSM is where both the stator core and rotor magnet of the motor rotate simultaneously.

Finally, to assess energy loss by the stator core of $\mathrm{PMSM} / \mathrm{G}$, after the stator core and the PM of PMSM/G rotor had been installed, the rotational speed degradation according to time was measured. The stator cores used in this experiment were a Si-steel core and an amorphous core. The Si-steel had a $0.1 \mathrm{~mm}$ thickness. The amorphous core had a $25 \mu \mathrm{m}$ ribbon thickness and an electrical resistivity of $130 \Omega \mathrm{m}$. The resistivity of the amorphous core is more than twice that of the Si-steel core. The high resistivity helps reduce the eddy current loss that rises sharply with increasing rotational speed. The tensile strength and hardness of the amorphous core were more than three times that of the Si-steel core. As shown in Fig. 2(b), the rotational speed degradation, changing core inner radius $R_{\text {is }}$ and outer radius $R_{\text {os }}$ size were measured. The following core sizes were used for the test: inner radius $R_{\text {is }}=30 \mathrm{~mm}$ and an outer radius of $R_{o s}=42 \mathrm{~mm}, 50$ $\mathrm{mm}$, and $62 \mathrm{~mm}$.

\section{Experimental Results}

The coefficient of friction was calculated to estimate the loss caused by magnetic flux friction in the SFES. The coefficient of friction [3] was $\mu=-[(\mathrm{dE} / \mathrm{dt}) / \mathrm{E}] \mathrm{v} / 2 \mathrm{~g}$, where $E$ is the rotational kinetic energy, $t$ is time, $v$ is velocity at the bearing perimeter, and $g$ is acceleration due to gravity.

Fig. 3 shows the coefficient of friction $\mu$ according to the rotational speed in the case of using a Si-steel core. In Fig. 3, the dots were the measured data and the lines were the curve fitting data. The coefficient of friction $\mu$ was linear with the rotational speed. Through curve fitting, the intercept was non-zero, which represents the coefficient of friction corresponding to hysteresis loss. The slope corresponded to the eddy current loss. $\mu$ was lower when

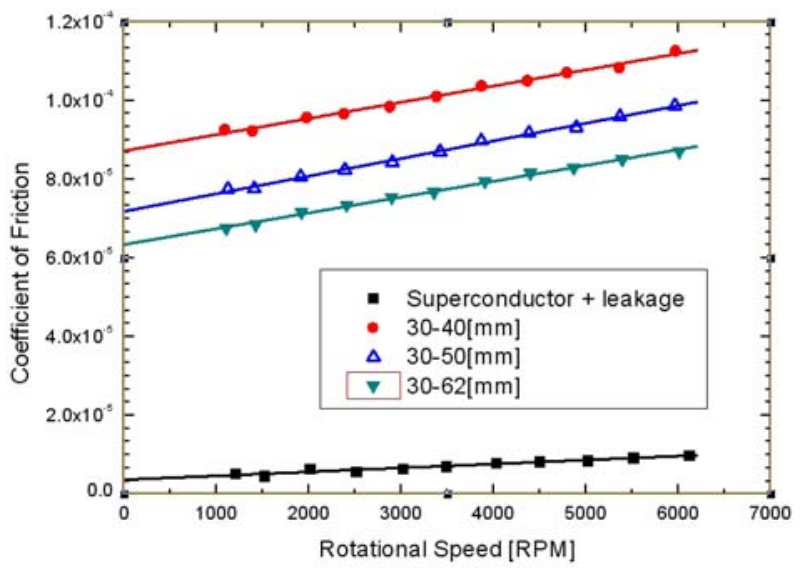

Fig. 3. Coefficient of friction according to the Si-steel size change including superconductor and leakage friction. (mark: experimental results, line: curve fitting).

outer radius was increased. The magnetic flux density decreased with increasing radius. The coefficient of friction $\mu$ of $30-40 \mathrm{~mm}, 30-50 \mathrm{~mm}$ and $30-62 \mathrm{~mm}$ was $8.7212 \times 10^{-5}, 7.18532 \times 10^{-5}$, and $6.34149 \times 10^{-5}$, respectively. It was confirmed that the outer radius needs to be increased to design a PMSM/S for SFES despite the increase in PMSM/G size. Magnetic friction in the case of using a Si-steel core was very high compared to that of SMB with leakage.

Fig. 4 shows the coefficient of friction $\mu$ according to rotational speed in the case of using an amorphous core. In Fig. 4, the dots are the measured data and the lines are the curve fitting data. This non-zero intercept is the coefficient of friction, which corresponded to hysteresis loss. The slope corresponded to the eddy current loss. $\mu$ was lower when the outer radius was increased but there was little difference. This was because the amorphous

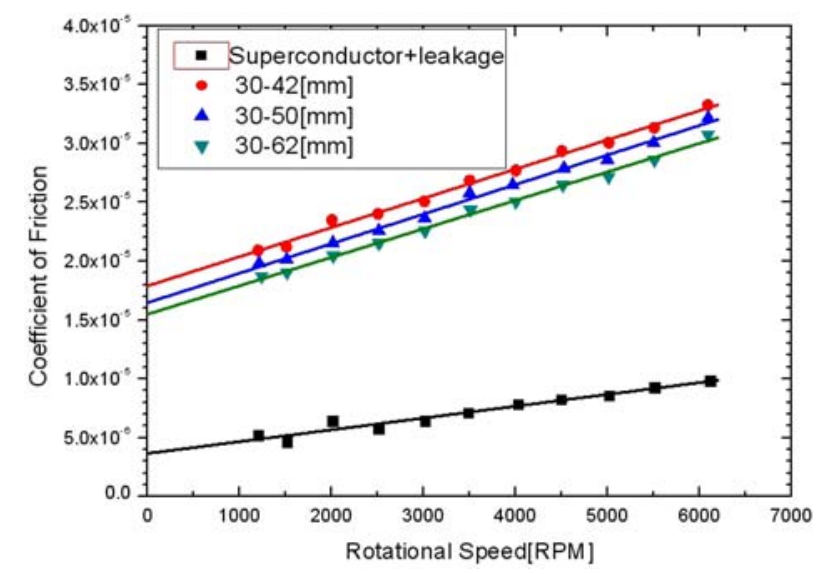

Fig. 4. Coefficient of friction according to the amorphous core size change including superconductor and leakage friction. (mark: experimental results, line: curve fitting). 


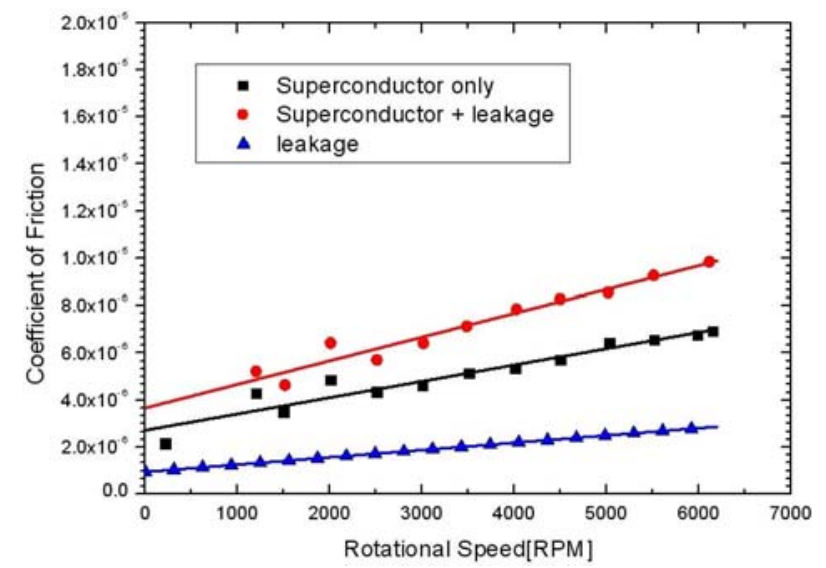

Fig. 5. Coefficient of friction by the superconductor and leakage flux density. (mark: experimental results, line: curve fitting).

core has little hysteresis loss. The coefficients of friction $\mu$ of 30-40 mm, 30-50 $\mathrm{mm}$ and 30-62 $\mathrm{mm}$ were 1.78965 $\times 10^{-5}, 1.6465 \times 10^{-5}$, and $1.5490 \times 10^{-5}$, respectively. Magnetic friction in the case of using an amorphous core was still higher than that of SMB with leakage.

Fig. 5 shows the coefficient of friction $\mu$ according to the rotational speed by the SMB and leakage flux. In Fig. 5, the dots are the measured data and the lines are the curve fitting data. The coefficients of friction $\mu$ of superconductor + leakage and superconductor were 3.66318 $\times 10^{-6}$ and $2.6804 \times 10^{-6}$, respectively. The leakage flux density in the metal part nearby a magnet of the PMSM was measured. The value was $2.5 \mathrm{mT}$. $\mu$ by this value was almost half of that of the SMB. Therefore, to reduce this value, an air gap of slotless type PMSM/G needs to be minimized or a conductor near the permanent magnet of

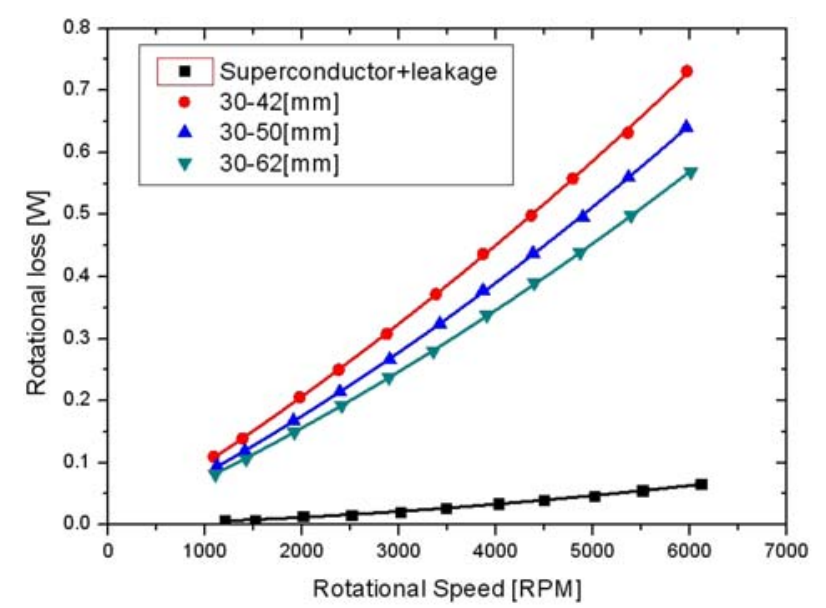

Fig. 6. Rotational loss per minute according to the rotational speed in the case of using a Si-steel core. (mark: experimental results, line: curve fitting).

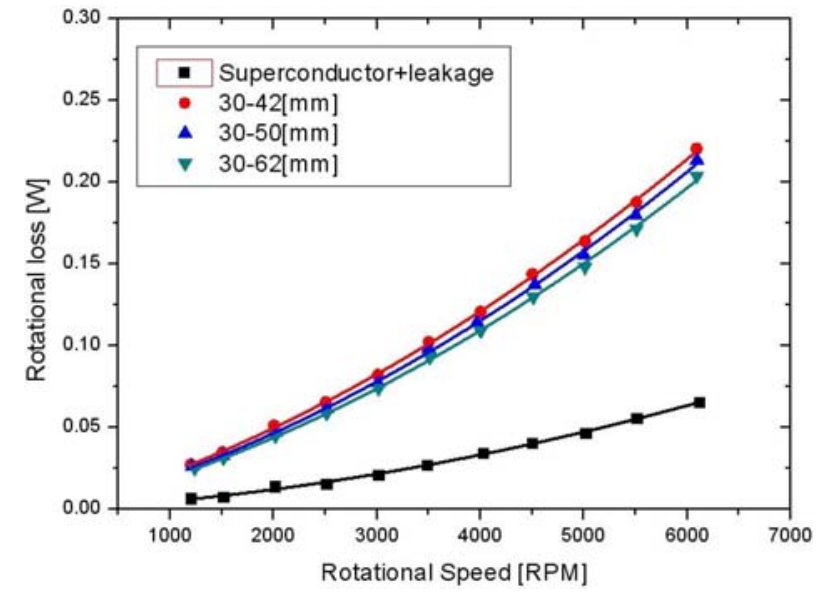

Fig. 7. Rotational loss per minute according to the rotational speed in the case of using amorphous core. (mark: experimental results, line: curve fitting).

the PMSM/G needs to be eliminated.

Figs. 6 and 7 show the results of rotational loss per minute in the case of using the Si-steel core and amorphous core. In the case of using an amorphous core, the rotational loss per minute was dramatically reduced. In Figs. 6 and 7, the rotational loss per minute for the Sisteel and amorphous core include superconductor and leakage loss. If the rotational loss by the superconductor and leakage are eliminated, the rotational loss by the amorphous core was approximately 2 times the loss of the superconductor and leakage. Moreover, the rotational loss by the Si-steel core was approximately $3 \sim 3.5$ times that of the superconductor and leakage.

\section{Conclusions}

In this paper, the axial type SFES system using the journal type superconductor bearings was manufactured with the PMSM/G. The coefficients of friction by the SMB, the Si-steel core and amorphous core of the PMSM/G, and leakage flux density were calculated using the spin down experiment of the SFES. The coefficients of friction decreased with increasing outer radius of the Si-steel core but were no affected by the outer radius of the amorphous core. These results were because the amorphous core showed little hysteresis loss. Therefore, in the case of using a Si-steel core to design the PMSM/S for the SFES, the outer radius needs be increased despite the increase in $\mathrm{PMSM} / \mathrm{G}$ size. On the other hand, in case of using the amorphous core to design the PMSM/G for the SFES, the outer radius does not need to be increased. To reduce friction loss by leakage flux density, air gap of the slotless type PMSM/G should be minimized or a conductor near 
the permanent magnet of the $\mathrm{PMSM} / \mathrm{G}$ should be eliminated. In the manufactured SFES, the rotational loss by the amorphous core was approximately 2 times the loss of the superconductor and leakage. Moreover, the rotational loss by the Si-steel core was approximately $3 \sim 3.5$ times that of the superconductor and leakage.

\section{Acknowledgment}

This study was supported by Ministry of Knowledge Economy, Republic of Korea.

\section{References}

[1] J. R. Hull, Supercon. Sci. Technol. 13, R1 (2000).
[2] Y. H. Han, J. R. Hull, S. C. Han, N. H. Jeong, T. H. Sung, and K. S. No, IEEE Trans. Appl. Supercond. 15, 2249 (2005).

[3] J. R. Hull, T. M. Mulcahy, and K. L. Uherka, IEEE Trans. Appl. Supercond. 5, 626 (1995).

[4] R. Shiraishi, K. Demachi, M. Uesaka, and R. Takahata, IEEE Trans. Appl. Supercond. 13, 2279 (2003).

[5] J. P. Lee, J. N. Jeong, Y. H. Han, S. C. Han, S. Y. Jung, B. J. Park, and T. H. Sung, IEEE Trans. Appl. Supercond. 19, 2087 (2009).

[6] J. P. Lee, B. J. Park, Y. H. Han, S. Y. Jung, T. H. Sung, IEEE Trans. Magn. 44, 4397 (2008).

[7] I. Masaie, K. Demachi, T. Ichihara and M. Uesaka, IEEE Trans. Appl. Supercond. 15, 2257 (2005).

[8] A. C. Day, J. R. Hull et al., IEEE Trans. Appl. Supercond. 13, 2179 (2003). 